ИЗВЕСТИЯ АКАДЕМИИ НАУК ЭСТОНСКОИ ССР. ФИЗИКА МАТЕМАТИКА

PROCEEDINGS OF THE ACADEMY OF SCIENCES OF THE ESTONIAN SSR. PHYSICS * MATHEMATICS

$1988,37,3$

\title{
РАСЧЕТ КОНТУРОВ БЕСФОНОННЫХ ЛИНИЙ ПРИМЕСНЫХ МОЛЕКУЛ В УСЛОВИЯХ ПЕРЕНОСА ЭНЕРГИИ
}

\author{
(Представил К. К. Ребане)
}

\section{Введение}

Молекулярные примеси в низкотемпературных матрицах характеризуются, как правило, спектрально неоднородным составом и поэтому однородные бесфононные линии (БФЛ) их электронно-колебательных спектров проявляются только при селективном (монохроматическом) возбуждении $\left[{ }^{1,2}\right]$. Влияние безызлучательного переноса электронных возбуждений на селективные спектры молекулярных систем исследовалось ранее [3, $\left.{ }^{3}\right]$. Рассматривался случай низких температур, когда средняя тепловая энергия $k T$ во много раз меньше неоднородной ширины и перенос энергии (ПЭ) происходит в основном путем необратимых процессов. При этом выводилось и численно решалось уравнение для спектральной диффузии при необратимом ПЭ и была найдена концентрационная зависимость интегральной интенсивности БФЛ флуоресценции.

В данной работе влияние ПЭ на БФЛ анализируется более подробно. Рассматриваются механизмы, которые приводят к изменению контура БФЛ в условиях ПЭ, в частности, к уширению ее. При этом кроме БФЛ флуоресценции рассматриваются соответствующие спектральные провалы в спектрах поглощения и возбуждения. Именно методом выжигания провала $\left[{ }^{2,5}\right]$ были измерены наиболее узкие, чисто электронные линии у молекулярных примесных систем (однородной шириной порядка $10 \mathrm{MГц).} \mathrm{Это,} \mathrm{в} \mathrm{частности,} \mathrm{означает,} \mathrm{что} \mathrm{вопрос} \mathrm{о}$ влиянии ПЭ на форму БФЛ вышел за рамки чисто академических задач. Отметим, что анализ контура бесфононных провалов в концентрированных примесных системах представляется не маловажным и с точки зрения многочисленных приложений метода выжигания провала.

В разделе 1 рассматриваем процессы ПЭ, в ходе которых рождается один или несколько матричных фононов. При низких температурах такие процессы эффективны в случае разности начального и конечного электронного состояния $\Delta E \gg k T\left[{ }^{6}\right]$, а роль сопряженных процессов с уничтожением фононов пренебрежимо мала. При возбуждении системы примесных центров монохроматическим светом происходит необратимый ПЭ от резонансно возбуждаемых центров (с частотами перехода в узкой спектральной области порядка однородной ширины БФЛ $\Gamma \ll k T)$ к более длинноволновым центрам. Имеет место известный эффект уменьшения средней времени жизни возбужденного состояния донорных (резонансных) центров [ $\left.{ }^{7}\right]$, а соответственно, и уширения БФЛ.

В разделе 2 рассматриваем двухфононные процессы, при которых $\Delta E$ определяется разностью частоты фононов, и которые эффективны 
при малых $\Delta E<k T$. Контур БФЛ определяется теперь спектральной диффузией в результате обратимого ПЭ. Отметим. что такая ситуация характерна для редкоземельных ионов в кристаллических матрицах и она подробно изучена для случая импульсного возбуждения $\left[{ }^{8}\right]$. Для молекулярных примесей, у которых времена жизни флуоресценцин существенно короче (наносекундные) и измерение резонансных БФЛ с временным разрешением относительно сложно, представляет интерес и случай стационарного возбуждения.

\section{1. Уширение БФЛ в результате необратимого переноса}

При монохроматическом возбуждении спектрально неоднородной системы невзаимодействующих примесных центров возникает резонансная линия флуоресценции с удвоенной однородной шириной $2 \Gamma_{0}$ $\left[{ }^{9}\right]$. В присутствии необратимого ПӘ от резонансно возбужденных примесей к однородной ширине БФЛ их прибавляется скорость ПЭ $V: \Gamma=\Gamma_{0}+V$. Для определения наблюдаемого контура БФЛ флуоресценции $I(\Delta v)$ надо провести усредненне БФЛ донорных центров с всевозможными окружностями акцепторов, имея в виду кроме вариаций пространственных конфигураций и их разный состав по частотам БФЛ. При отсутствии корреляции между пространственным и частотным распределением центров $I(\Delta v)$ можно выразить в виде:

$$
I(\Delta v) \sim \int_{0}^{\infty} d \Phi(V) \frac{1}{1+V \tau} \frac{\Gamma_{0}+V}{\Delta v^{2}+\left(\Gamma_{0}+V\right)^{2}},
$$

где $\Phi(V)$ является распределением примесей по полной скорости ПЭ ( $\tau$ - время жизни возбужденного состояния в отсутствии ПЭ). При диполь-дипольном ПЭ на хаотически расположенные акцепторы

$$
\Phi(V)=\pi^{-1 / 2} q(V \tau)^{-3 / 2} \exp \left(-\frac{q^{2}}{V \tau}\right)
$$

$\left[{ }^{10}\right]$, где в спектрально неоднородном случае параметр $q$ зависит от частоты возбуждения $\left[{ }^{4}\right]$ :

$$
q(v)=2,56 C C_{0}^{-1}(v), \quad C_{0}^{-1}(v)=\int_{0}^{v} d P\left(v^{\prime}\right) r_{0}^{3}\left(v, v^{\prime}\right) .
$$

Здесь $C$ - концентрация примесей, $P(v)$ - функция неоднородного распределения $(Ф Н \mathrm{P}), r_{0}\left(v, v^{\prime}\right)-$ т. н. радиус переноса от донора с частотой БФЛ $v$ к акцептору с частотой $v^{\prime *}$.

На рис. 1 изображены БФЛ флуоресценции, описываемые формулой (1) на пределе низких температур, когда процессы фазовой релаксации выморожены $\left(\Gamma_{0}=\tau^{-1}\right)$ и влияние ПЭ на контур БФЛ наиболее сильно. Концентрационное уширение линии в этом случае сопряжено с эффектом уменьшения средней длительности флуоресценции $\bar{\tau}\left[{ }^{7}\right]$. На основе (1) можно показать, что интегральная интенсивность БФЛ пропорциональна выходу флуоресценции $\eta\left(\right.$ см. $\left.\left[{ }^{3,4}\right]\right)$, а пиковая интенсивность - произведению $\eta \cdot \bar{\tau}$. Следовательно, можно ожидать, что ширина БФЛ $\bar{\Gamma}$ на полувысоте будет расти пропорционально величине $\bar{\tau}^{-1}$. Расчет концентрационной зависимости ширины $\bar{\Gamma}$ показывает, что

* Вероятность элементарного акта переноса определена как $w=\tau-1 \chi^{2} r_{0}{ }^{6} / r^{6}$, где $r$ расстояние между донором и акцептором, $\chi$ - ориентационный фактор (см. [7]). 
Надо отметить, что в случае спектрального провала от конщентрации не будет зависеть предельная глубина (насыщенного) провала, a аналогом уменьшения интенсивности БФЛ флуоресценции служит замедление скорости выжигания. В начальной стадии выжигания она замедляется в центре провала пропорционально величине $\eta \cdot \bar{\tau}$, а по площади провала как $\eta$. Подчеркнем, что последнее обстоятельство было использовано для определения параметров ПЭ в замороженных растворах хлорофилла [ $\left.{ }^{11}\right]$.

Если провал детектируется не в спектре поглощения, а по флуоресценции (например, в спектре возбуждения), то форма БФЛ несколько изменяется (см. рис. 2). Теперь относительный квантовый выход выступает в формуле (1) дважды, т. е. в ней вместо $(1+V \tau)^{-1}$ стоит $(1+V \tau)^{-2}$. Асимптотическая связь между средней длительностью флуоресценции и шириной провала теперь следующая: $\bar{\Gamma}_{e} / 2=$ $=0,49 \cdot \bar{\tau}^{-1}$.

\section{2. Спектральная диффузия при квазирезонансном переносе}

ПЭ между примесями, частоты БФЛ которых находятся в узкой спектральной области вокруг частоты монохроматического возбуждения $v_{e}$, осуществляется двухфононными процессами $\left[{ }^{6}\right]$, причем при выполнении условия $\Delta E<k T$ последние являются обратимыми (т. е. возбуждения могут возвращаться на исходный центр). Спектральную диффузию в результате таких процессов можно описать балансным уравнением $\left[{ }^{12}\right]$, которое в случае стационарного возбуждения выражается в виде:

$$
n(v)=I_{0} x\left(v_{e}-v\right) \tau+\int_{0}^{\infty} d P\left(v^{\prime}\right)\left[W\left(v^{\prime}, v\right) n\left(v^{\prime}\right)-n(v) W\left(v, v^{\prime}\right)\right],
$$

где $n(v)$ - средняя относительная населенность возбужденного состояния, $x\left(v_{e}-v\right)-$ БФЛ поглощения примесных центров с частотой перехода $v, I_{0}$ - интенсивность возбуждения. Эффективную вероятность переноса от примесных центров с частотой БФЛ $v$ к центрам с частотой БФЛ $v^{\prime} W\left(v, v^{\prime}\right)$ можно найти аналогичной процедурой усреднения, развитой в $\left[{ }^{4,13}\right]$ для описания необратимого ПЭ в системе хаотически расположенных примесей. Основой последней является приближение, согласно которой окружности (расположение окружающих примесей) двух фиксированных в пространстве примесей некоррелированы. При диполь-дипольном взаимодействии $\left(r_{R}\left(v, v^{\prime}\right)\right.$ - радиус переноса) окончательный результат усреднения следующий:

$W\left(v, v^{\prime}\right)=\frac{\pi^{2}}{3}\left[1+\frac{1}{2 \sqrt{3}} \ln (2+\sqrt{3})\right] \operatorname{Cr}_{R}^{3}\left(v, v^{\prime}\right)\left\{1+\exp \left[\frac{\hbar\left(v^{\prime}-v\right)}{k T}\right]\right\}^{-1 / 2}$.

В случае процессов ПЭ рамановского типа (два фонона взаимодействуют только с одним из примесных центров, между которыми происходит скачок электронного возбуждения) радиус переноса можно определить как

$$
r_{R}^{6}(v, v)=\frac{D}{\pi} \frac{\Gamma_{0}}{\left(v-v^{\prime}\right)^{2}+\Gamma_{0}^{2}}
$$

$\left[{ }^{6}\right]$, где $D-$ независящий от частот множитель, а однородная ширина БФЛ $\Gamma_{0}$ не зависит от ПЭ. По причине медленной сходимости интег- 
ралов уравнения (4), под которыми стоит квадратный корень от функции $(6)$, важно иметь в виду реальное обрезание крыльев $r_{R}^{6}\left(v, v^{\prime}\right)$ при большой частотной расстройке от центра линии $\left[{ }^{14}\right]$. Согласно $\left[{ }^{15}\right]$, лоренцовый контур БФЛ наблюдается до величины расстройки $\Delta$, при которой становится заметным изменение интенсивности фононного крыла (порядок величины $\Delta \sim 100$ ГГц).

Уравнение (4) решалось нами путем замены его системой алгебраических уравнений, с помощью которых описали ПЭ между 139 участками в интервале $\left(v_{e}-\Delta\right) \div\left(v_{e}+\Delta\right)$. $P(v)$ считалась в этом интервале константной, а экспоненциальный член в выражении (5) - равным единице. Точный учет последнего при условии $ћ \Delta \leqslant k T$ привел бы лишь к малой асимметрии линии флуоресценции на ее далеких крыльях. Параметром при расчете служила относительная концентрация $C / C_{R}$,

где

$$
C_{R}^{-1}=\int_{0}^{\infty} d P\left(v^{\prime}\right) r_{R}^{3}\left(v_{e}, v^{\prime}\right)=2 \sqrt{\frac{D \Gamma_{0}}{\pi}} \ln \left|\frac{2 \Delta}{\Gamma_{0}}\right| P\left(v_{e}\right)
$$

Как видно из рис. 3, где изображена зависимость $n\left(v_{e}-v\right)$ от концентрации в случае $\Delta=7 \Gamma_{0}$, населенность перераспределяется от центральной части линии на крылья, причем средний пик практически не уширяется. Увеличение спектральной ширины ПЭ $\Delta$ приводит в основном к более пологому распределению $n(\Delta v)$ на крыльях. Что касается центральной части $n(\Delta v)$, то, например, при $\Delta=20 \Gamma_{0}$ и $C / C_{R}=1$ она отличается от соответственной кривой рис. 3 лишь несколько меньшим константным фоном под ней (фон уменьшается примерно на $15 \%$ от пиковой величины $n(\Delta v))$. Наблюдаемая БФЛ (а также малый спектральный провал) определяется сверткой $n(v \Delta)$ и однородной БФЛ и концентрационная зависимость ее контура практически такая же, как y $n(\Delta v)$.

Надо отметить, что рассмотренная картина спектральной диффузии весьма сходна с таковой в случае ПЭ, вероятность которого не зависит от $\Delta E=\hbar\left(v^{\prime}-v\right)\left(r_{R}^{3}\left(v, v^{\prime}\right)=r_{R}^{3}\right)$. Последний осуществляется при $\Delta E<k T$, когда два фонона, разность энергий которых компенсирует изменение энергии электронного возбуждения при переносе, взаимодействуют с разными примесными центрами $\left[{ }^{6}\right]$. Согласно модельным

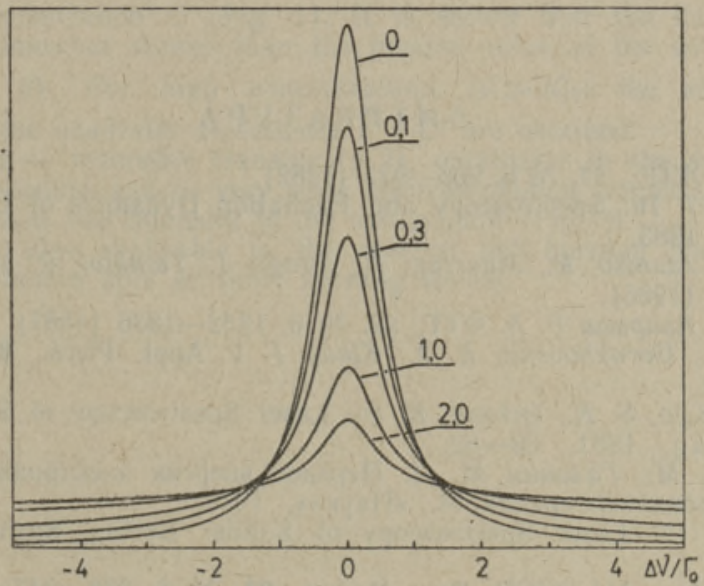

Рис. 3. Спектральное распределение населенности возбужденного состояния $n(\Delta v)$ в случае обратимого переноса энергии рамановскими процессами. У кривых указаны величины относительной концентрации примесей $C / C_{R}$. 
расчетам $\left[{ }^{8,16}\right]$, такой механизм приводит к перераспределению насёленностей от центрального пика шириной $2 \Gamma_{0}$ к фону с контуром ФНР. В данном случае является возможным точное определение относительной интенсивности центрального пика $\chi_{R}$, которая в приближении некоррелированных окружений дается формулой:

$$
\chi_{R}\left(C r_{R}^{3}\right)=\left(1+3,21 \cdot C r_{R}^{3}\right)^{-1} .
$$

Интересно отметить, что выражение (8) опюсывает в использованном приближении и концентрационную зависимость относительной анизотропии флуоресценции в случае однородно уширенной линии. Сравнение ее с соответствующими зависимостями известных теорий концентрационного тушения поляризацции $\left[{ }^{17}\right]$ показывает, что результаты совпадают с точностью $10 \%$ при концентрациях $\quad C \leqslant r_{R}^{-3}$.

В заключение сравним эффективность рассмотренных в разделе 1 и 2 процессов ПЭ. В случае сильного неоднородного уширения (системы с неупорядоченными матрицами) в принципе должны иметь место оба механизма. Оценка величин характеристических концентраций $C_{0}$ и $C_{R}$ показывает, что для примесей со средними стоксовыми потерями обратимый ПЭ в спектре практически не наблюдается. В этом случае $C_{0} / C_{R}<0,1$, а кроме того, эффективная вероятность $W\left(v, v^{\prime}\right)$ (формула (5)) в присутствии спектрально удаленных акцепторов уменьшается: она умножается на фактор $\sqrt{\eta\left(C / C_{0}\right)}$, где $\eta-$ относительный выход флуоресценции (см. [ $\left.\left.{ }^{4}\right]\right)$. Эффект обратимого ПЭ становится преобладающим лишь в случае малого неоднородного уширения (ширина ФНР во много меньше частоты Дебая) и/или в случае предельно слабых стоксовых потерь. Таким образом, хотя, с одной стороны, нами рассматривались два частных случая проявления ПЭ в форме БФЛ $\left(\Gamma_{0}=\tau^{-1}\right.$ в разделе 1 и $\Gamma_{0} \gg \tau^{-1}$ в разделе 2$)$, с другой, эти случаи весьма характерны для систем с большим и малым неоднородным уширением соответственно.

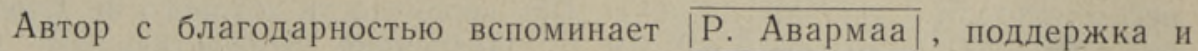
ценные замечания которого способствовали выполнению данной работы.

\section{Л И Т Е Р А Т Р А}

1. Ребане К. К. ЖПС, 37, № 6, 906-922 (1982).

2. Personov, R. I. In: Spectroscopy and Excitation Dynamics of Condensed Molecular Systems, 1983 .

3. Avarmaa, R., Jaaniso, R., Mauring, K., Renge, I., Tamkivi, R. Mol. Phys., 57, № 3, 605-621 (1986).

4. Яанисо Р. В., Авармаа Р. А. ФТТ, 29, № 6, 1832-1836 (1987)

5. Rebane, L. A., Gorokhouskii, A. A., Kikas, J. V. Appl. Phys., B29, № 2, 235-250 (1982).

6. Holstein, T., Lyo, S. K., Orbach, R. In: Laser Spectroscopy of Solids. Berlin, Springer Verlag, 1981, 39-82.

7. Агранович В. М., Галанин М. Д. Перенос энергии электронного возбуждения в конденсированных средах. М., «Наука», 1978.

8. Huber, $D$. L. In: Laser Spectroscopy of Solids. Berlin, Springer Verlag, 1981, 83-111.

9. Авармаа Р. Изв. АН ЭССР. Физ. Матем., 23, № 3, 238-247 (1974).

10. Максимов М. 3., Розман Н. М. Опт. и спектр., 12, № 5, 606-609 (1962).

11. Яанисо Р., Тамкиви Р., Ренге И., Мауринг К. Изв. АН ЭССР. Физ. Матем., 36 № 2, 189-192 (1987).

12. Motegi, N., Shionoya, S. J. Luminescence, 8, № 1, 1-17 (1973). 
13. Бодунов E. Н., Мальциев В. А. ФТТ, 23, № 4, 1087-1092 (1981); 24, № 12, $3654-3662(1982)$.

14. Буритейн А. Н. Автометрия, № 6, 72-90 (1978).

15. Осадько Н. С. ФТТ, 14, № 10, 2927-2934 (1972).

16. Бодунов E. Н., Малышев В. А. ФТТ, 27, № 12, 3642-3652 (1985).

17. Knox, R. S. Physica, 39, 361-386 (1968).

Ннститут физики

Академии наук Эстонской ССР
Поступила в редакцию

9/VII 1987

\section{R. JAANISO}

\section{LISANDIMOLEKULIDE FOONONVABADE JOONTE KUJU ARVUTUS ENERGIA OLEKANDE TINGIMUSTES}

Teoreetiliselt on uuritud energia ülekande mõju madalatemperatuurilises maatriksis paiknevate lisandimolekulide foononvabadele joontele. Spektraalselt mittehomogeense lisandisüsteemi fluorestsentsijoone või spektraalsälgu kuju leidmiseks on tehtud keskmistamine üle lisandite juhusliku ruumijaotuse diipol-diipol-ülekande korral. Eraldi on vaadeldud pöörduva ja pöördumatu ülekande mõju. Viimane on efektiivne suure spektraalse mittehomogeensusega süsteemides ja viib madalal temperatuuril foononvabade joonfe laienemisele. Suhteliselt kitsas spektraalpiirkonnas toimuva pöörduva ülekande pōhiilminguks on joone intensiivsuse ümberjaotumine fooni.

\section{R. JAANISO}

\section{THE CALCULATION OF THE SHAPE OF ZERO-PHONON LINES IN IMPURITY MOLECULES ON ENERGY TRANSFER}

The influence of the excitation energy transfer on the zero-phonon lines of impurity molecules in a low-temperature matrix is treated theoretically. The model takes into account the dipol-dipol transfer in a spectrally and spatially disordered impurity system. After averaging over the distances and dipol orientations the lineshape is determined by the formula (1) in case of irreversible transfer (energy gap between donor and acceptor $\Delta E \gg k T$ ) or by balance equation (4) with «spectral transfer» rate $W\left(v, v^{\prime}\right)(5)$ in case of reversible transfer $(\Delta E<k T)$.

The irreversible mechanism (p.1) is effective in the systems with large inhomogeneous spectra and leads to the broadening of the zero-phonon line with the increase of the impurity concentration $C$ (Fig. 1). It is shown that the linewidth (fwhm) $\bar{\Gamma}$ increases with $C$ somewhat slower than the inverse value of the average fluorescence lifetime $\bar{\tau}^{-1}$ (Fig. 2). For high concentrations $\left(C>2 C_{0}\right)$ the asymptotic relation $\bar{\Gamma} / 2=0.72 \cdot \bar{\tau}^{-1}$ and the quadratic dependence $\bar{\Gamma} \sim C^{2}$ are obtained.

The main effect of reversible transfer (p.2), obtainable in the systems with small inhomogeneous broadening and/or very weak electron-phonon coupling, is the redistribution of the zero-phonon line intensity to the background (Fig. 3).

The results are also applicable to the spectral hole-burning phenomenon (to the shape of the zero-phonon hole at short burning times). 